Discussion Paper No. 09-025

\title{
Entwicklung und Ungleichheit
} von Fähigkeiten: Anmerkungen aus ökonomischer Sicht

Friedhelm Pfeiffer

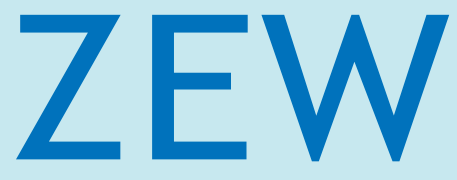

Zentrum für Europäische Wirtschaftsforschung $\mathrm{GmbH}$

Centre for European Economic Research 
Discussion Paper No. 09-025

\title{
Entwicklung und Ungleichheit von Fähigkeiten: Anmerkungen aus ökonomischer Sicht
}

\author{
Friedhelm Pfeiffer
}

Download this ZEW Discussion Paper from our ftp server:

ftp://ftp.zew.de/pub/zew-docs/dp/dp09025.pdf

Die Discussion Papers dienen einer möglichst schnellen Verbreitung von neueren Forschungsarbeiten des ZEW. Die Beiträge liegen in alleiniger Verantwortung der Autoren und stellen nicht notwendigerweise die Meinung des ZEW dar.

Discussion Papers are intended to make results of ZEW research promptly available to other economists in order to encourage discussion and suggestions for revisions. The authors are solely responsible for the contents which do not necessarily represent the opinion of the ZEW. 


\section{Das Wichtigste in Kürze}

Nirgends sonst im ökonomischen Handeln fallen Kosten und Nutzen im Zeitablauf und aufgeteilt nach Investoren und Nutznießern so eklatant auseinander wie bei Bildungsinvestitionen. Ein bedeutender Teil der für die Entwicklung von Fähigkeiten relevanten Investitionen wird mit Beginn der Empfängnis bis ins Schulalter getätigt. Ein erheblicher Teil der aus der Entwicklung von Fähigkeiten entstehenden Erträge fällt im Erwachsenenalter an. Die Investoren und diejenigen, die die Erträge erhalten, sind vielfach nicht identisch. Zu Beginn des Lebens sind, außer dem Lernenden selbst, die Eltern entscheidend, ab dem Schulalter kommen Bildungseinrichtungen hinzu. Im Erwachsenenalter profitieren von den Erträgen der Lernende, seine Familie sowie die Gemeinschaft, beispielsweise über höhere Steuern.

In dem vorliegenden Beitrag wird argumentiert, dass in der sozialen Realität die Bildungsungleichheit im Vorschulalter eine wichtige Ursache für die Ungleichheit von Fähigkeiten und Kompetenzen auch im Schulalter und im Erwerbsleben ist. In der Kindheit werden in der Interaktion zwischen Kind und Erwachsenem die Kapazitäten aufgebaut (oder nicht aufgebaut), welche eine nachhaltige Voraussetzung für den Schul- und Arbeitsmarkterfolg schaffen. Die Unterschiede in der Verfügbarkeit von adäquaten emotionalen Ressourcen (,Bodenfreiheit“, elterliches „Kontingenzverhalten“, ...) in der Kindheit entfalten eine prägende Kraft, die im späteren Leben in der sozialen Wirklichkeit kaum mehr aufgeholt werden kann. Im Schulalter kommen finanzielle Restriktionen durch Armut in der Familie noch hinzu.

Während beispielsweise 74\% der Kinder ein Gymnasium besuchen, die ohne organische und psychosoziale Risiken bei der Geburt aufwachsen konnten, trifft das nur für 15\% der Kinder zu, die mit beiden Risiken aufgewachsen sind. Eine um 10\% bessere elterliche Fürsorge im Säuglingsalter geht mit einem um 5,5\% höheren Entwicklungsstand des Kindes einher. Bis zum Schulalter sinkt dieser Wert bereits auf unter 2\%. Trotz Schulpflicht, hohen Bildungsausgaben und hoher Wirtschaftskraft hat demnach die Ungleichheit der elterlichen Fürsorge vor dem Schulalter ihren schicksalhaften Charakter für die Kompetenzentwicklung noch keineswegs verloren. Neben dem erheblichen individuellen Leid, das mit solchem Schicksal verbunden sein kann, entstehen der Gesellschaft dadurch signifikante Kosten, unter anderem im Gesundheits- und Sozialsystem. Medizinische Forschungen deuten beispielsweise darauf hin, dass Vernachlässigung und Gewalt in der Kindheit die Anfälligkeit für Depressionen, aber auch für Infektionskrankheiten im Erwachsenenalter erhöhen.

Um dies zu ändern, bleibt es eine vordringliche Aufgabe auch der Bildungspolitik, den Zugang zu einer angemessenen emotionalen Fürsorge von Anfang an weiter zu verbessern. Darüber hinaus ist es notwendig, den betroffenen Kindern bis ins Jugendalter altersgemäß und individuell zur Seite zu stehen. Für Jugendliche und Erwachsene, die während der Kindheit nur unzureichend gefördert wurden, sollten Maßnahmen erforscht werden, die helfen können, vielfältige Benachteiligungen aus der Kindheit im späteren Lebenszyklus zu bewältigen. 


\section{Nontechnical Summary}

There is no other economic activity, where costs and benefits are as far apart in time and where investors and beneficiaries are as detached as is the case for investments in human capital. The largest part of investments in capacity formation takes place from the moment of conception until school age, while returns (employment, earning, and occupation) are realized mostly in adulthood. During childhood parents are the most important investors. Apart from the parents, starting with school age, educational institutions invest in academic competencies like reading and math. In adulthood the individual benefits from returns to human capital investments, as well as her family and the society, e.g. through higher taxes.

This study argues that inequality in investments during pre-school age is one of the most important reasons for the inequality of outcomes in school and adult life. In early childhood, capacities are built (or not built), mainly in the family, through interactions between children and parents. Differences in the availability of adequate emotional interaction and resources ("floor freedom", "parental responsiveness", ...) during childhood are formative in the sense that a later catch-up is hard to achieve. During the transition from primary to secondary education financial constraints due to economic poverty in the family and imperfect credit markets for adolescent children create an additional barrier to investments into higher education.

For instance, $74 \%$ of the children which grow up without organic and psychosocial risks at birth attend the highest secondary school track, compared to only $15 \%$ of the children who grew up with both risks. A $10 \%$ improvement in the quality of parental care in infancy is associated with a 5.5\% improvement in child development. The strength of this relationship is reduced to less than $2 \%$ once upon school age is reached. The inequality of parental care before school age therefore remains critical fro competence formation, despite compulsory schooling, high educational expenditures and substantial wealth in modern economies. In addition to the individual distress associated with adverse child outcomes, the latter causes significant costs to society e.g. to the education and the health care systems. Medical research for instance suggests that parental neglect and violence during childhood increases the susceptibility to depression, and inflammations during adulthood. Furthermore, it increases the probability of academic failure.

To foster human capital, policy should further improve the availability of adequate parental assistance for disadvantaged children of preschool age. In addition, it is necessary to support and accompany these children until adolescence in an ageappropriate and individual way. For adults, who have not received adequate investments during pre-school age compensating measures are required, whose design and effectiveness are subject to further investigation. 


\title{
Entwicklung und Ungleichheit von Fähigkeiten:
}

\section{Anmerkungen aus ökonomischer Sicht}

\author{
Friedhelm Pfeiffer \\ Zentrum für Europäische Wirtschaftsforschung (ZEW), Mannheim
}

20. Mai 2009

\section{Zusammenfassung:}

Nirgends sonst im ökonomischen Handeln fallen Kosten und Nutzen im Zeitablauf und aufgeteilt nach Investoren und Nutznießern so eklatant auseinander wie bei Bildungsinvestitionen. In dem vorliegenden Beitrag wird argumentiert, dass in der sozialen Realität die Bildungsungleichheit im Vorschulalter eine der wichtigsten Ursachen für die Ungleichheit von Fähigkeiten und Kompetenzen auch im Schulalter und im Erwerbsleben ist. Für benachteiligte Kinder scheint somit die Bildungsungleichheit vor dem Schulalter bei uns, ebenso wie in anderen Ländern mit hohen Bildungsausgaben und hoher Wirtschaftskraft ihren schicksalhaften Charakter noch keineswegs verloren zu haben. Um dies zu ändern, bleibt es eine vordringliche Aufgabe auch der Bildungspolitik, den Zugang zu einer angemessenen emotionalen Fürsorge von Anfang an weiter zu verbessern. Darüber hinaus ist es notwendig, den betroffenen Kindern bis ins Jugendalter altersgemäß und individuell zur Seite zu stehen.

Schlüsselworte: Anfangsbedingungen, Elterliche Fürsorge, Fähigkeiten, Investitionen in Humankapital, Lebenszyklus.

JEL-Klassifikation: D87, I12, I21, J13

\section{Danksagung:}

Der Autor dankt der Leibnizgemeinschaft, Bonn für die Förderung des Projektes “Nichtkognitive Fähigkeiten: Erwerb und ökonomische Konsequenzen” im Rahmen des Paktes für Forschung und Innovation 2008. Ferner möchte ich mich bei Anja Achtziger, Ute Bayer, Jo Blanden, Dorothea Blomeyer, Katja Coneus, Peter Drewek, Philipp Eisenhauer, Wolfgang Franz, Michael Gebel, Johannes Gernandt, Peter Gollwitzer, James Heckman, Fabian Kosse, Stefan Küpper, Manfred Laucht, Andrea Mühlenweg, Pia Pinger, Winfried Pohlmeier, Karsten Reuß, Julia Schäfer, Jürgen Schupp, C. Katharina Spieß, Emma Tominey und Gert Wagner für die Zusammenarbeit in den letzten Jahren bedanken. Der vorliegende Beitrag ist eine gekürzte Fassung meines Vortrags bei der internationalen Tagung „Bildungsungleichheit revisited“ des Zentrums für Schul- und Bildungsforschung der Martin Luther Universität Halle-Wittenberg im Oktober 2008. Für verbleibende Fehler und Unzulänglichkeiten bin ich alleine verantwortlich.

\section{Adresse:}

Friedhelm Pfeiffer, Zentrum für Europäische Wirtschaftsforschung (ZEW), Postfach 103443, D-68034 Mannheim. Tel.: +49-621-1235-150, E-mail: pfeiffer@zew.de. 


\section{Einleitung}

Ausdauer und Leistungsintensität im Handlungsablauf, Motivation, sprachliche, logische und motorische Fähigkeiten, Gedächtnisleistungen ebenso wie die Fähigkeit zur Verarbeitung von Informationen sind im Schul- und Arbeitsalltag von erheblicher Bedeutung. Die Forschung geht davon aus, dass diese Fähigkeiten durch geeignete Maßnahmen ausbaubar sind. Die bildungsökonomische Forschung wirkt an der Beantwortung der praktischen Frage mit, wann, wie und in welchem Umfang dies aus ökonomischer Sicht geschehen sollte. Dabei steht im folgenden Beitrag der investive Charakter von Bildung im Vordergrund, der sich aus einer Abwägung von Kosten und Nutzen im Lebenszyklus ergibt.

Nirgends sonst im ökonomischen Handeln fallen Kosten und Nutzen im Zeitablauf und aufgeteilt nach Investoren und Nutznießern so eklatant auseinander wie bei Bildungsinvestitionen. Ein bedeutender Teil der für die Entwicklung von Fähigkeiten relevanten Investitionen wird mit Beginn der Empfängnis bis ins Schulalter getätigt. Ein erheblicher Teil der aus der Entwicklung von Fähigkeiten entstehenden Erträge fällt im Erwachsenenalter an. Die Investoren und diejenigen, die die Erträge erhalten, sind vielfach nicht identisch. Zu Beginn des Lebens sind, außer dem Lernenden selbst, die Eltern entscheidend, ab dem Schulalter kommen Bildungseinrichtungen hinzu. Im Erwachsenenalter profitieren von den Erträgen der Lernende, seine Familie sowie die Gemeinschaft, beispielsweise über höhere Steuern.

Für die aus der Entwicklung und aus Bildungsinvestitionen resultierenden Fähigkeiten hat sich in der Ökonomie der Begriff Humankapital eingebürgert. Als Humankapital wird die Bewertung menschlicher Fähigkeiten und Handlungen bezeichnet, die in Tauschprozessen in der sozialen Realität zustande kommt, innerhalb der Familie, der Gemeinschaft, eines Unternehmens oder auf einem (Arbeits-)Markt. Der Zusammenhang zwischen den Ressourcen, die zum Erwerb der Fähigkeiten aufgewendet werden, den Inputfaktoren, und dem Niveau der damit erreichbaren Fähigkeiten, dem Output, wird als Produktionsfunktion bezeichnet, die aus der Technologie des Erwerbs von Fähigkeiten (Cunha und Heckman (2007, 2009)) abgleitet werden kann. Umstritten ist, ob Bildungsinvestitionen im Lebenszyklus immer einen vergleichbaren Ertrag ermöglichen oder ob es entwicklungsabhängig unterschiedliche Erträge geben kann. Heckman (2007) argumentiert, dass die ersten Lebensjahre, inklusive der Entwicklung im Mutterleib, die Phase der Kapazitätsbildung im Lebenszyklus darstellt, in der Investitionen besonders ertragreich seien. Allgemeine Problemlösungsfähigkeiten, schulische und berufliche Kompetenzen würden von der Kapazitätsbildung in der Kindheit bestimmt, im Sinne des Sprichworts: „Was Hänschen nicht lernt, lernt Hans nimmermehr“. Ohne eine ausreichende Kapazitätsbildung seien spätere Investitionen ansonsten kaum mehr ertragreich.

Dahinter steht die Vorstellung einer kumulativen Natur der Entwicklung von Fähigkeiten, eines aufeinander aufbauenden Entwicklungsprozesses, in dem die Entfal- 
tung in der jeweils nächsten Stufe vom Zustand des Erreichten aus der vorhergehenden Entwicklungsstufe abhängt. ${ }^{1}$ Bildungssysteme sind in aller Regel ebenfalls auf einer solchen Vorstellung aufgebaut. Jedoch würden (siehe Heckman (2000)) die Konsequenzen der eminenten Rolle der Kapazitätsbildung in der frühen Kindheit noch zu wenig in Reformen des Bildungssystems umgesetzt. ${ }^{2}$ Insbesondere für benachteiligte Kinder, deren Eltern nur ungenügende Investitionen bereitstellen, können Schulen keine ausreichende Kompensation mehr bewirken. Vielmehr sei, sofern es nicht gelinge, zusätzliche öffentliche Mittel bereitzustellen (was angesichts des Grades der öffentlichen Verschuldung bezweifelt werden könne), eine (moderate) Verlagerung von öffentlichen Bildungsressourcen in den frühen Lebenszyklus erforderlich. Viele Untersuchungen zur Rolle der Kindheit für die Humankapitalbildung (zusammenfassend Heckman $(2007,2008)$ ) beziehen sich auf US-Daten. Ökonomische Analysen mit Längsschnittstudien für Deutschland, die kognitive, motorische und nichtkognitive Fähigkeiten von der Geburt an enthalten, zusammen mit den wichtigsten familiären und institutionellen Einflussfaktoren, sind noch Mangelware.

Ziel des vorliegenden Beitrags ist es, die Investitionsströme in Deutschland auf Basis einer Lebenszyklusbetrachtung gedanklich zu strukturieren und Folgerungen für die optimale Entwicklung von Fähigkeiten zu diskutieren. Zur empirischen Verdeutlichung werden Untersuchungsergebnisse von Blomeyer et al. (2008, 2009) zum Zusammenhang zwischen organischen und psychosozialen Geburtsbedingungen, der elterlichen Fürsorge und der Entwicklung grundlegender Fähigkeiten, sowie sozialer und schulischer Kompetenzen in den ersten elf Lebensjahren, ebenso herangezogen wie Modelle der Humankapitalbildung im Lebenszyklus von Pfeiffer und Reuß (2008a,b). Der Kindheitsmultiplikator, der Kapazitätsaufbau in der Kindheit für die lebenslange Humankapitalbildung, wird quantitativ aufgearbeitet.

Die Ergebnisse deuten darauf hin, dass die Bildungsungleichheit im (Vor-)Schulalter eine der zentralen Ursachen von Ungleichheiten ist. Sie stehen somit, wenig überra-

1 Die Vorstellung findet sich auch in der Psychologie, siehe unter anderem Braun und Stern (2007), Holodynski et al. (2008). Auch Sigmund Freuds Theorie der Persönlichkeit liegt die Vorstellung einer Entwicklung in Phasen zugrunde, wobei Freud vor allem die frühe Kindheit hervorgehoben hat. Erikson (1959) unterscheidet, aufbauend auf Freud, acht Phasen der Persönlichkeitsentwicklung im Lebenszyklus, von denen jede ihre spezifische Bedeutung hat. Für die Persönlichkeitsentwicklung sind nach Erikson alle Phasen gleich wichtig.

2 Das folgende Zitat von Johann Wolfgang von Goethe (1795, Wilhelm Meisters Lehrjahre, Zweites Buch, neuntes Kapitel) deutet darauf hin, dass der Konflikt schon älter zu sein scheint: “Aber“, versetzte Wilhelm, „wird das Genie sich nicht selbst retten, die Wunden, die es sich geschlagen, selbst heilen?“ „Mitnichten“, versetze der andere, „Oder, wenigstens nur notdürftig; denn niemand glaube die ersten Eindrücke der Jugend überwinden zu können. Ist er in einer löblichen Freiheit, umgeben von schönen und edlen Gegenständen, in dem Umgang mit edlem Menschen aufgewachsen, haben ihn seine Meister das gelehrt, was er zuerst wissen mußte, um das übrige leichter zu begreifen, hat er gelernt, was er nie zu verlernen braucht, wurden seine Handlungen so geleitet, daß er das Gute künftig leichter und bequemer vollbringen kann, ohne sich irgend etwas abgewöhnen zu müssen, so wird dieser Mensch ein reineres, vollkommneres und glücklicheres Leben führen als ein anderer, der seine ersten Jugendkräfte im Widerstand und im Irrtum zugesetzt hat. Es wird soviel von Erziehung gesprochen und geschrieben, und ich sehe nur wenig Menschen, die den einfachen, aber großen Begriff, der alles andere in sich schließt, fassen und in die Ausführung übertragen können.“ 
schend, im Einklang mit einer umfangreichen Literatur ${ }^{3}$ (unter anderem Amor (2003), Black et al. (2007), Braun und Stern (2007), Coneus und Spieß (2008), Heckhausen und Heckhausen (2006), Heckman (2007), Holodynski et al. (2008), Laucht et al. (1997, 2004), O’Conell und Sheikh (2008), van den Berg et al. (2006), Sodian und Koerber (2008)). Die Unterschiede in der Verfügbarkeit von emotionalen Ressourcen in der Kindheit entfalten eine die Entwicklung prägende Kraft. Das (Klein-) Kind kann nicht unmittelbar von besseren materiellen Ressourcen profitieren, sondern nur von der Verfügbarkeit höherwertiger emotionaler Ressourcen. Im Schulalter kommen finanzielle Restriktionen durch Armut in der Familie hinzu. Für viele benachteiligte Kinder hat daher auch in Ländern mit hoher Wirtschaftskraft die Bildungsungleichheit vor dem Schulalter ihren schicksalhaften Charakter noch nicht verloren. Um dies zu ändern, ist der Zugang zu emotionaler Fürsorge von Anfang an zu verbessern. Die betroffenen Kinder müssen darüber hinaus bis ins Jugendalter weiter gefördert werden. Im Beitrag werden bildungspolitische Maßnahmen im Vorschulalter mit Interventionen in der Grundschule und beim Übergang von der primären zur sekundären Bildung aus einer ökonomischen Sicht verglichen.

Wenngleich die in der aktuellen Forschung zusammengetragene Evidenz zur Rolle der (frühen) Kindheit für die Entwicklung und die Ungleichheit von Fähigkeiten eindrucksvoll ist, und bildungspolitische Antworten erfordert, bleiben aus wissenschaftlicher Sicht offene Fragen bestehen. Zwar zeigen die Ergebnisse, dass Investitionsdefizite während der vorschulischen und schulischen Lebensphase im späteren Lebenszyklus in der sozialen Realität kaum mehr wettgemacht werden. Dabei ist der Bezug zur „sozialen Realität“ wichtig. Empirische Ergebnisse werden in einem konkreten Familien-, Gesellschafts- und Schulkontext, eben der sozialen Realität, in der das Kind lebt, gemessen. Untersucht wird der Entwicklungsprozess einer Population von Kindern von der Geburt bis zum Schulalter in einer bestimmten sozialen Realität, die zu einer messbaren Evolution der Ungleichheit von Fähigkeiten beigetragen hat. Diese soziale Realität wirkt tatsächlich für viele Kinder und Jugendliche schicksalhaft. Auch in dem vorliegenden Beitrag werden eine Reihe prägender Faktoren, darunter organische und psychosoziale Geburtsrisiken, fehlendes mütterliches Kontingenzverhalten, Gewalt und Ausgrenzung in der Familie, herausgearbeitet.

Der empirische Kindheitsmultiplikator kann jedoch möglicherweise keine universelle Gültigkeit beanspruchen, in dem Sinne, dass Fähigkeiten nach der Kindheit nicht mehr veränderbar sind. Eine andere soziale Realität, beispielsweise in Form einer Schule, die einen größeren Teil von Ressourcen für benachteiligte Kinder einsetzt, oder die mit einer stärker individuell strukturierten Interaktion Kindern den fehlenden kompetenten Anderen ersetzt, kann neue Erkenntnisse zu Tage fördern. Zukünftige Studien, die die Nachhaltigkeit von kompensierenden, experimentell variieren-

3 "The most valuable of all capital is that invested in human beings; and of that capital the most precious part is the result of the care and influence of the mother”, Alfred Marshall (1890, Principles of Economics, VI.IV.11). 
den Investitionen im Kindergarten- und Schulalter für in ihrer Kindheit benachteiligte Kinder untersuchen, sind notwendig, um diese Forschungslücke zu schließen.

\section{Die Bildung von Fähigkeiten im Lebenszyklus}

Im Rahmen dieses Beitrags ist es nicht möglich, alle Aspekte der Humankapitalbildung mit gleicher Priorität abzuhandeln. Im Vordergrund steht das Thema der Entwicklung von Fähigkeiten über den Lebenszyklus, unabhängig davon, ob Investitionen von Bildungseinrichtungen im Rahmen formalisierter Lernsituationen getätigt werden, oder im Rahmen informeller Lernprozesse, zusammen mit Freunden und in der Familie. Erst diese Gesamtschau ermöglicht eine Würdigung der Architektur des staatlichen Bildungswesens aus ökonomischer Sicht. ${ }^{4}$

Menschen haben Anlagen zur Entwicklung einer immensen Vielfalt von Fähigkeiten, Kenntnissen und Kompetenzen. Diese können im Wirtschaftsleben, sei es in einer selbständigen oder in einer abhängigen Erwerbstätigkeit, ebenso wie im gesellschaftlichen und privaten Leben zur Entfaltung kommen und vielfältige, zum Teil subjektive (Glück), zum Teil objektiv messbare Erträge (Lohn) erzielen. Aufbauend auf den biologischen, pädagogischen und psychologischen Grundlagen untersuchen Bildungsökonomen den optimalen Einsatz knapper Ressourcen zum Erwerb dieser Fähigkeiten im Lebensverlauf, sowie die Anreizstrukturen der Investoren. Diese Forschung wirkt an der Beantwortung der praktischen Frage mit, welche Fähigkeiten durch gezielte Bildungsmaßnahmen verbessert werden können und in welcher Höhe diese Investitionen wann erfolgen sollten.

Trotz Bildungsexpansion und weitgehend unabhängig von der Finanzierung und Regelung der vielfältigen nationalen Bildungssystemen sind die Bildungsressourcen in der sozialen Wirklichkeit ungleich verteilt, sowohl in einem Querschnitt von Lernenden zu einem Zeitpunkt wie auch über den gesamten Lebenszyklus eines Individuums. Die (frühe) Kindheit liegt in den Händen von Müttern, Vätern oder anderen Betreuungspersonen. Aufgrund von Unterschieden in den Kompetenzen und Möglichkeiten der etwa 8,8 Millionen Familien (mit Kindern unter 18 Jahren, im Jahre 2006, www.destatis.de) in Deutschland, ausreichende Investitionen bereitzustellen, sind bereits erhebliche Unterschiede in der Fähigkeitsentwicklung der Kinder zu erwarten. Auch der Zeithorizont spielt eine wichtige Rolle. Es gibt einen erheblichen zeitlichen Unterschied zwischen Bildungsinvestitionen und deren Erträgen. Das ist ein grundsätzliches, der Produktion von Fähigkeiten innewohnendes Problem, das möglicherweise eine wichtige Ursache für die Vielfalt realer Gesellschafts- und Bildungssysteme ist.

Lernen fängt im Mutterleib an und ist ein Teil unseres biologischen Erbes. Bereits der „kompetente Säugling“ (Sodian und Koerber (2008:83)) investiert einen erhebli-

4 Die Rolle der allgemeinen und der beruflichen Bildung im technischen Wandel wird im Beitrag nicht explizit angesprochen, vgl. dazu unter anderem Ludwig und Pfeiffer (2006). 
chen Teil seiner Zeit und seiner Möglichkeiten in die eigene Entwicklung. Auch nach der Ablösung aus der körperlichen Symbiose mit dem Mutterleib kann sich der Säugling noch nicht alleine weiterentwickeln. Er benötigt den „kompetenten Anderen“ (Holodynski et al. (2008: 93)). Es setzt eine Phase intensiver Interaktion zwischen dem kompetenten Säugling und dem kompetenten Anderen ein. Diese frühe Eltern-Kind Interaktion gilt vielfach als „Wiege des Handelns“ (Heckhausen und Heckhausen (2006:402)). Da die elterlichen Ressourcen, die die Entwicklung befördern, darunter insbesondere eine dem Entwicklungsstadium entsprechende emotionale Unterstützung („Bodenfreiheit“, „mütterliches Kontigenzverhalten“, etc., Heckhausen und Heckhausen (2006:403f.)), unterschiedlich verteilt sind, ist der Zugang zu diesem wichtigen Input in die Produktion von Fähigkeiten zwischen Kindern bereits von frühester Kindheit an verschieden. ${ }^{5}$ Da zudem die Ressourcen in der Regel und relativ zur Nachfrage nach Unterstützung seitens ihrer Kinder begrenzt sind, werden die kompetenten Anderen Prioritäten setzen, unter anderem durch die Wahl des Kindergartens oder der Schule.

Neben dem „kompetenten Anderen“ steht dem Lernenden im Schulalter die Schule zur Verfügung, wobei das Zutrittsalter von der Ausgestaltung des Bildungswesens abhängt, ebenso wie dessen Wirkungen (siehe Mühlenweg und Puhani (2009)). Auch die Schule stellt keinen homogenen, für alle Schüler gleichartig wirkenden Lernkontext her. Schulen sind, selbst bei einheitlich festgelegten Lehrplänen, heterogen (siehe unter anderem Beck und Klieme (2006)). Analysen deuten darauf hin, dass Schulen in Deutschland die familiär bedingte Ungleichheit von Fähigkeiten nicht verstärken, sondern in der Summe (moderat) kompensierend wirken (unter anderem Mühlenweg (2008)), in dem Sinne, dass die Ungleichheit nicht zunimmt. Kramarz et al. (2008) kommen mit Daten aus England zu dem Schluss, dass Schülerleistungen empirisch in erster Linie vom familiären Hintergrund erklärt werden und, in geringerem Maße, vom Lernumfeld. Hanushek und Wößmann (2008) finden, auf der Basis einer Panelstudie mit 50 Industrie- und Entwicklungsländern, dass höhere kognitive Fähigkeiten (gemessen mit Hilfe von internationalen Schülerleistungstests), die durch Schulen beeinflusst werden, zum Wirtschaftswachstum beitragen.

Eine häufige, in staatlichen Bildungsprogrammen seit der Französischen Revolution genannte Zielvorgabe öffentlicher Einrichtungen ist es, Kinder unabhängig von ih-

5 Benabou und Tirol (2004) untersuchen Konsequenzen frühkindlicher Interaktionsprozesse für die Willenskraft und die gegenwartsbezogene Zeitpräferenzen, siehe auch Ainslie (2001). Unter dem Stichwort „Eltern-Kind Konflikte“ gibt es zu diesem grundlegenden Aspekt der Entwicklung ebenso eine lesenswerte biologische Literatur, siehe Wells (2003). Nach Danesi et al. (2007) erhöhen Gewalt und Misshandlungen in der Kindheit die Anfälligkeit für Depressionen und Infektionen im Erwachsenenalter. Es ist umstritten, ob und wie dies kompensiert werden kann. Zur Qualität eines kompetenten Anderen gehört dessen Präsenz. Partielle Maßnahmen, wie beispielsweise eine hypothetische (drei- bis vierstündige) Kindergartenpflicht, können bei einem 24 Stunden Tag kaum einheitlich positiv wirken, da das Kind im Tagesablauf 20 bis 21 Stunden nicht im Kindergarten ist. 
rem familiären Hintergrund in gleicher Weise ${ }^{6}$ zu fördern. Hinzu kommen weitere Ziele, etwa dass Kinder individuell, ihrem Entwicklungsstand gemäß, zu fördern seien. Kinder mit Lernschwierigkeiten erhalten bei dieser Zielvorgabe einen anderen Unterricht als beispielsweise Hochbegabte. Da auch den Schulen nur begrenzte Ressourcen zur Verfügung stehen, können nicht alle Ziele gleich gut erreicht werden, und es erfolgt eine Prioritätensetzung seitens der Schulen, die sich nicht zuletzt in der Zusammensetzung der Fächer und der Unterrichtsform manifestiert.

Bis ins Erwachsenenalter braucht der Lernende zusätzlich zur Schule den kompetenten Anderen. Nach Gollwitzer (1999) (siehe auch Gollwitzer et al. (2008) sowie die dort zitierte Literatur) führen verbesserte Methoden der Selbstregulation in einer Reihe von herausfordernden Lebenslagen (,in the face of hardship“) zu besseren Ergebnissen („einfache Vorsätze, große Wirkungen“). Der kompetente Andere wäre in diesem Sinne die Person, die dem Lernenden hilft, seine selbstregulatorischen Fähigkeiten zu verbessern. Investitionen in die stetige Entwicklung von Kompetenzen werden sowohl vom Lernenden, von seiner Familie wie auch von der Schule getätigt. Diese drei Gruppen von Beteiligten unterscheiden sich hinsichtlich ihrer Vorstellungen, wie auch im Hinblick auf die zur Verfügung stehenden Möglichkeiten, gezielte Investitionen zu tätigen. Die bildungsökonomische Forschung steht daher vor der Notwendigkeit, die unterschiedlichen Investitionsbeiträge zu erfassen und deren partielle Erträge abzuschätzen. Dies ist keine einfache Aufgabe, weil die Investitionen in einem bestimmten Zeitpunkt und über den Lebenszyklus komplementär oder substitutiv sein können.

Eine staatliche Ausweitung der Schul- oder Kindergartenpflicht könnte einige Eltern oder Schüler zu einer Reduktion ihrer ansonsten vorgesehenen Bildungsinvestitionen veranlassen. In dem Fall würden trotz Schulpflicht die Investitionen in der Summe nicht zunehmen oder zumindest nicht im Ausmaß der Erweiterung der Schulpflicht. Andere Eltern werden ihre eigenen Bildungsanstrengungen noch erhöhen, um die Vorteile, die mit einer guten Schulausbildung verbunden sein können, auszuschöpfen. Für die Gruppe dieser Kinder würde die erweiterte Schulpflicht dazu führen, dass die gesamten Investitionen die Summe aus schulischen und elterlichen Investitionen vor deren Einführung, übersteigen. Anreizprobleme ergeben sich daraus, dass die Erträge einer erfolgreichen Förderung von Fähigkeiten nicht notwendigerweise oder nicht im angemessenen Umfang den unterschiedlichen Investoren zufallen. Dies kann in der Summe zu einer Unterinvestition, zu einer suboptimalen Aufteilung der Investitionen, oder beidem, führen.

Die Humankapitalforschung beschäftigt sich mit den ökonomischen Erträgen, die unter anderem durch eine höhere Partizipation am Erwerbsleben sowie eine höhere

6 „Ich verlange, .... daß ... alle Kinder, ohne Unterschied und Ausnahme, gemeinschaftlich erzogen werden sollen auf Kosten der Republik, und dass alle unter dem heiligen Gesetz der Gleichheit dieselbe Kleidung, dieselbe Nahrung, denselben Unterricht, dieselbe Sorgfalt erhalten." Michel Lepeletier (1794): Plan einer Nationalerziehung (nach Michael, Berthold/Heinz-Hermann Schepp (1993), Die Schule in Staat und Gesellschaft, S. 88). 
Produktivität erzielt werden können. Die Bewertung der Produktivität wird auf firmeninternen Arbeitsmärkten, durch kollektive und individuelle Lohnverhandlungen, sowie durch Angebot und Nachfrage auf Arbeitsmärkten vorgenommen. Die durch Investitionen in die Fähigkeiten und Kompetenzen gestiegene höhere Produktivität kommt (zumindest zum Teil) dem Individuum in Form eines höheren Arbeitsverdienstes zu Gute.

Gebel und Pfeiffer (2009) untersuchen die Evolution der Bildungsrendite von 1984 bis 2006 in Deutschland. Demnach liegt die durchschnittliche Bildungsrendite in der Gruppe der Bildungsteilnehmer bei einem Wert von etwa 6\%. Ein zusätzliches Jahr in Vollzeitausbildung ist mit einem um 6\% höheren Arbeitsverdienst verbunden. ${ }^{7}$ 6\% sind aus ökonomischer Sicht eine passable Rendite, die auch für die Bildungspolitik eine wichtige Orientierungsmarke darstellen könnte. Die Probleme der Trennung der Investitionen durch die formale Ausbildung von den gleichzeitig stattfindenden individuellen und familiären Investitionen sind Gegenstand laufender Studien. Man kann nicht ausschließen, dass der Schätzwert überhöht oder zu niedrig ist. Auch ist die Bildungsrendite individuell verschieden. Bei einem Durchschnittswert von $6 \%$ ist sie für fast 25\% der Arbeitnehmer negativ und für fast 25\% der Arbeitnehmer 10\% oder höher (Maier et al. (2004)).

Wenn der Einzelne für seine Investitionen selbst Sorge tragen kann, wäre Anreizkompatibilität zu erwarten. Möglicherweise nimmt mit zunehmender Qualität der Kompetenzen in der Bevölkerung darüber hinaus auch die volkswirtschaftliche Produktivität oder der in einer Gesellschaft in der Summe erzielbare Wohlstand zu. Sei es, dass mit mehr Fähigkeiten externe positive Effekte in Produktionsprozessen einhergehen, oder sei es, dass es gelänge, das gesellschaftliche Zusammenleben durch Vermeidung negativer externer Effekte (unter anderem weniger Kriminalität) zu verbessern. Falls es dafür Evidenz gäbe, wäre eine Unterinvestition in Bildung im Schulalter die wahrscheinliche Folge und damit auch aus ökonomischer Sicht eine Rationalität öffentlicher Bildungsinvestitionen in diesem Lebensabschnitt gegeben.

Studien zum Thema externe Erträge von Investitionen im Schulalter kommen zwar nicht zu eindeutigen Ergebnissen (siehe Ciccone und Peri (2006), Moretti (2004) unter anderem). Das kann angesichts der Identifikationserfordernisse nicht überraschen. Jedoch könnte ein Ergebnis aus den Untersuchungen mit der Mannheimer Risikokinderstudie hilfreich sein, und der Forschung zu diesem Thema neue Hypothesen liefern. Nach Blomeyer et al. (2008) prognostizieren die grundlegenden kognitiven, motorischen, und nichtkognitiven Fähigkeiten im Vorschulalter die Qualität von Freundschaften im Schulalter. Wichtige Bestimmungsgründe von mehr sozialer Integration scheinen somit bereits mit dem erreichten Niveau von kognitiven, moto-

7 Die Bildungsrendite gibt den Ertrag („Arbeitsentgelt“) von formaler Bildungsbeteiligung in Abhängigkeit von der Dauer des Besuchs von (Hoch-)Schulen in Prozent an. Gebel und Pfeiffer (2009) weisen darauf hin, dass diese so gemessene Bildungsrendite keine Naturkonstante ist. Sie hängt unter anderem von Angebot und Nachfrage auf dem Arbeitsmarkt ab, scheint aber in Deutschland über die Jahre nicht besonders stark zu schwanken. Nach einem Rückgang in den neunziger Jahren hat die Bildungsrendite in den letzten Jahren wieder (leicht) zugenommen. 
rischen, und nichtkognitiven Fähigkeiten im Vorschulalter zusammenzuhängen. Dies würde bedeuten, dass vermeintliche soziale Vorteile von mehr Schulbildung, externe Effekte, ihre tiefere Verankerung in vorteilhaften Entwicklungsprozessen bereits im Vorschulalter haben. Das Ergebnis könnte die nicht einheitliche Evidenz zu externen Effekten von Humankapital im Arbeitsleben erklären. Der Kindheitsmultiplikator für die Humankapitalbildung würde somit noch immer unterschätzt. Im folgenden Abschnitt werden Auswertungen aus der Mannheimer Risikokinderstudie vorgestellt, die psychometrische Tests zu kognitiven, motorischen und nichtkognitiven Fähigkeiten von der Geburt bis ins Jugendalter enthalten, und die Abschätzungen des Kindheitsmultiplikators ermöglichen.

\section{Geburtsbedingungen und Kompetenzentwicklung}

Die Ergebnisse stammen aus einer Kooperation des Zentralinstituts für seelische Gesundheit (ZI) und dem Zentrum für Europäische Wirtschaftsforschung (ZEW) in Mannheim (siehe ausführlich Blomeyer et al. (2008, 2009)). Als Datengrundlage dient die Mannheimer Risikokinderstudie, eine Längsschnittstudie zur Erforschung der Auswirkungen von Geburtsrisiken im Lebensverlauf, die am Zentralinstitut für Seelische Gesundheit in Mannheim durchgeführt wird (Laucht (2005)). Diese Untersuchung, die im Jahre 1986 begann, umfasst 384 erstgeborene Kinder mit deutsch sprechenden Eltern in der Rhein-Neckar-Metropolregion. Die Kinder wurden bei der Geburt nach der Schwere organischer und psychosozialer Risiken ausgewählt. Zu den organischen Risiken zählen unter anderem Geburtskomplikationen, Frühgeburten und (sehr) niedriges Geburtsgewicht. Zu den psychosozialen Risiken zählen unter anderem eine frühe Elternschaft (die Eltern waren zum Zeitpunkt der Geburt unter 18 Jahre, oder die Beziehung der Eltern dauerte zum Zeitpunkt der Empfängnis weniger als sechs Monate), mangelnde Bildung der Eltern, sowie (erhebliche) Dissonanzen in der Beziehung der Eltern. In jeder Dimension wurden drei Kategorien, kein, moderates, hohes Risiko gebildet, sodass die anfängliche Risikomatrix neun Felder enthält (Tabelle 1).

Die in Tabelle 1 dargestellten Ergebnisse verdeutlichen, dass eine interpersonelle Ungleichheit der Fähigkeiten bereits sehr früh messbar ist. Dies gilt gleichermaßen für die kognitiven, motorischen wie auch die nichtkognitiven Fähigkeiten. Jedoch scheinen die Unterschiede, die sich durch die Zugehörigkeit zu einer der neun Risikozellen ergeben, spezifisch für die Fähigkeiten zu sein. Die organischen Geburtsrisiken scheinen einen deutlichen Zusammenhang mit den kognitiven und motorischen Fähigkeiten aufzuweisen, wohingegen die psychosozialen Geburtsrisiken unmittelbar stärker mit den nichtkognitiven Fähigkeiten assoziiert sind. Weiterhin verdeutlichen die Werte, dass sich die beiden Risiken kumulieren und sich im Zeitablauf gegenseitig verstärken. Das Geburtsrisiko setzt sich somit aus der Summe der organischen und psychosozialen Einzelrisiken zusammen (Laucht (2005)). Bis zum Alter von 11 Jahren, dem letzten Zeitpunkt in der Mannheimer Risikokinderstudie, 
zu dem die kognitiven, motorischen und nichtkognitiven Fähigkeiten der Kinder erhoben werden, sind die Gruppenunterschiede in den Zellen der Risikomatrix gewachsen.

Tabelle 1: Grundlegende kognitive, motorische und nichtkognitive Fähigkeiten im Alter von 3 Monaten und 11 Jahren, in Abhängigkeit von den organischen und psychosozialen Geburtsbedingungen (Gruppenmittelwerte, Standardabweichung in Klammern)

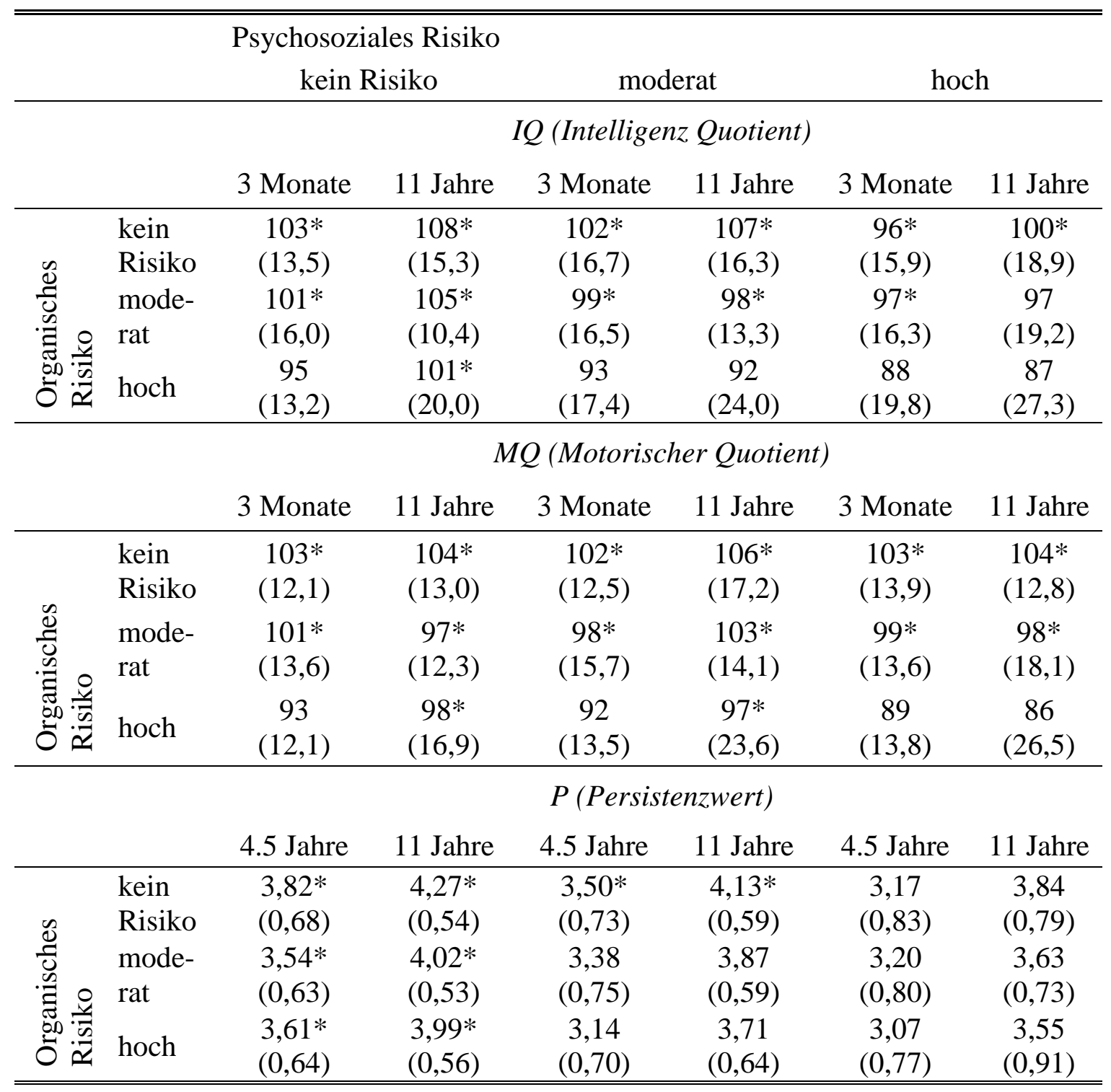

Werte entnommen aus Blomeyer et al. (2008, Tabelle A3). Der IQ und MQ wurden auf den Mittelwert 100 und die Standardabweichung normiert; das Ergebnis des Persistenzwerts variiert zwischen 1.0, 1.1, ... (niedrig) und 5.0 (hoch); *statistisch signifikante Differenz des Gruppenmittelwertes zur höchsten Risikogruppe zum 5\% Niveau.

Der Zusammenhang zwischen organischen und psychosozialen Geburtsbedingungen und dem Schulerfolg wie auch über die Qualität von Hobbys und Freundschaften im 
Schulalter wird in Tabelle 2 dargestellt. Während 74\% der Kinder ein Gymnasium besuchen, die ohne organische und psychosoziale Risiken bei der Geburt aufwachsen konnten, trifft das nur für 15\% der Kinder zu, die mit beiden Risiken aufgewachsen sind. Bei den Schulnoten in der Grundschule im Alter von acht Jahren hat die Studie zudem Unterschiede bis zu einer ganzen Note gefunden. Dabei zeigte sich, dass die psychosozialen Risiken für deutlich schlechtere Schulnoten verantwortlich sind, als die organischen.

Beispielsweise beträgt der mittlere Unterschied im IQ im Alter von 11 Jahren zwischen der Gruppe der Kinder mit maximaler Ausprägung beider Risiken und der Gruppe von Kindern ohne jegliches Risiko 21. Dieser hohe Wert verdeutlicht die erheblichen Konsequenzen organischer und psychosozialer Geburtsrisiken. Ein ähnlich großer Unterschied im IQ wurde auch zwischen den in Großbritannien adoptierten Kindern aus rumänischen Kinderheimen und einer Gruppe von innerhalb Großbritanniens adoptierten Kindern gefunden (siehe Beckett et al. (2006)). Die Kinder in den rumänischen Heimen wuchsen in den ersten Monaten ihres Lebens unter besonders schwierigen Entwicklungsbedingungen und (fast) ohne Betreuung durch kompetente Andere auf.

Tabelle 2: Schulnoten, Hobbys und Freundschaften im Alter von 8 Jahren, sowie höherer Schulbesuch im Alter von 11 Jahren in Abhängigkeit von den organischen und psychosozialen Geburtsbedingungen

\begin{tabular}{|c|c|c|c|c|}
\hline \multicolumn{5}{|c|}{ Psychosoziales Risiko } \\
\hline & & kein Risiko & moderat & hoch \\
\hline \multicolumn{5}{|c|}{ Schulnoten in Lesen ${ }^{a)} /$ Hobbys $^{b} /$ Freundschaften $^{b)}$ im Alter von 8 Jahren } \\
\hline \multirow{3}{*}{ 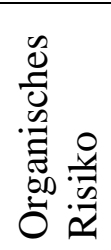 } & $\begin{array}{l}\text { kein Risi- } \\
\text { ko }\end{array}$ & $2,0 * / 5,1 * / 4,8 *$ & $2,2 * / 4,9 * / 4,6 *$ & $2,3 / 4,4 / 4,6^{*}$ \\
\hline & moderat & $2,2 * / 5,0 * / 4,5^{*}$ & $2,4 / 4,4 * / 4,5^{*}$ & $2,8 / 4,1 / 4,4$ \\
\hline & hoch & $2,1 * / 4,9 * / 4,8 *$ & $2,4 / 4,3 / 4,4$ & $2,8 / 3,9 / 4,0$ \\
\hline
\end{tabular}

Schulbesuch Gymnasium / Realschule / Andere ${ }^{\text {c) }}$ im Alter von 11 Jahren (in \%)

\begin{tabular}{|c|c|c|c|c|}
\hline & $\begin{array}{l}\text { kein Risi- } \\
\text { ko }\end{array}$ & $74 * / 24 * / 02 *$ & $77 * / 09 * / 14 *$ & $43 / 21 * / 36$ \\
\hline 苘 & moderat & 45 / 40* / 15* & 38 / 38* / 34* & 33 / 23 / 44 \\
\hline 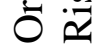 & hoch & $54 * / 23 * / 23 *$ & $27 / 38$ / 45 & $15 / 28 / 67$ \\
\hline
\end{tabular}

Werte entnommen aus Blomeyer et al. (2008, Tabelle 1, Tabelle A8). a) Schulnoten von 1 (sehr gut) bis 6 (ungenügend); b) Hobbys und Freundschaften wurden auf der Basis von Experteneinschätzungen gemessen, wobei die Werteskala von 1.0 (schlecht) bis 7.0 (sehr gut) reicht; c) Haupt, Förder- and Waldorfschule; *statistisch signifikante Differenz des Gruppenmittelwertes zur höchsten Risikogruppe zum 5\% Niveau. 
Auch bei den Hobbys und der Qualität der Freundschaften, die ein Kind hat, zeigen sich erhebliche Unterschiede in Abhängigkeit von der Zugehörigkeit zu einer der Risikozellen bei der Geburt, wobei die Assoziation zwischen psychosozialen Risiken und den sozialen Kompetenzen ausgeprägter zu sein scheint. In der Gruppe der Kinder ohne jegliches psychosoziales Risiko scheint eine Zunahme des organischen Risikos nicht mit einer Verschlechterung der Werte von Hobbys und Freundschaften einherzugehen. Diese Befunde belegen, dass sich Wirkungen von Geburtsrisiken nicht generalisieren lassen. Für jede Ergebnisgröße, seien es grundlegende Fähigkeiten oder schulische Kompetenzen, sind spezifische Resultate zu erwarten.

\section{Die Qualität der elterlichen Fürsorge und die Kompetenz- entwicklung}

Die oben erörterte Entwicklung der Gruppenmittelwerte von grundlegenden Fähigkeiten und den weiteren schulischen und sozialen Kompetenzen bis zum Jugendalter ist noch unvollständig. Eltern wenden auch nach der Geburt erhebliche Mittel für die Entwicklung ihrer Kinder auf, deren Bedeutung von Heckhausen und Heckhausen (2006), Heckman (2007), Holdynski et al. (2008) und anderen aufgezeigt wurde. In diesem Abschnitt möchte ich die Ergebnisse der ökonometrischen Analyse von Blomeyer et al. (2009) vorstellen, die erstmals für Deutschland den Beitrag der Qualität der elterlichen Fürsorge für die „Produktion“ von Fähigkeiten bis ins Jugendalter quantifiziert haben (siehe Schaubild 1). Die Quantifizierung des elterlichen Inputs für die Fähigkeiten des Kindes geschieht, technisch gesprochen, in der Form einer partiellen Elastizität. Der Wert der partiellen Elastizität gibt an, um wie viel Prozent sich die betrachtete Fähigkeit verbessert, wenn die Qualität des elterlichen Inputs um ein Prozent zunimmt, bei Konstanz der übrigen Einflussfaktoren. Blomeyer et al. (2009) gehen davon aus, dass die partielle Elastizität, die sich aus der „Produktionsfunktion“ von Fähigkeiten ergibt, für alle Kinder die gleiche ist.

In Schaubild 1 wird der Schätzwert der partiellen Elastizität in fünf Entwicklungsphasen (Säuglingsalter (3 Monate), Kleinkindalter (2 Jahre), Vorschulalter (4,5 Jahre), Grundschulalter (8 Jahre), Schulalter (11 Jahre)) jeweils für kognitive (gemessen mit dem Intelligenzquotienten, IQ), motorische (gemessen mit dem motorischen Quotienten, $M Q$ ) und nichtkognitiven Fähigkeiten (gemessen mit der Ausdauer im Handlungsablauf, Persistenz, $P$ ) angegeben. Schätzwerte der partiellen Elastizität unter 0,20 erweisen sich zum 5\% Niveau als nicht signifikant. Schaubild 1 verdeutlicht, dass die Qualität der elterlichen Fürsorge einen statistisch signifikanten und positiven Zusammenhang mit den kognitiven und nichtkognitiven Fähigkeiten hat, der jedoch im Entwicklungsverlauf unterschiedlich ausfällt.

Die partielle Elastizität weist bei den kognitiven Fähigkeiten im Vorschulalter einen hohen Wert von bis zu 0,55 auf. Auch ab dem Schulalter bleibt die Elastizität positiv und ist mit einem Schätzwert von 0,18 nicht niedrig. Zum 5\% Niveau kann jedoch 
die Nullhypothese (der Wert ist nicht von null verschieden) nicht verworfen werden. Die fehlende statistische Signifikanz könnte mit der niedrigen Fallzahl von 364 Beobachtungen zusammenhängen. In jedem Fall ist die partielle Elastizität im Vorschulalter hoch, danach niedrig. Vor allem im Vorschulalter trägt daher die Qualität der elterlichen Fürsorge zur Verbesserung der kognitiven Fähigkeiten bei. Im Alter zwischen 5 und 8 Jahren geht deren Bedeutung deutlich zurück. Während im Vorschulalter eine um 1\% verbesserte Qualität der elterlichen Fürsorge einen um bis zu 0,55\% höheren Wert des IQ zur Folge hat, sinkt die Verbesserung danach auf unter $0,2 \%$. Diesen Schätzungen folgend trägt die Ungleichheit in der Verfügbarkeit von elterlicher Fürsorge in den ersten vier bis fünf Lebensjahren zur Entwicklung der Ungleichheit der kognitiven Fähigkeiten bei.

Schaubild 1: Die partielle Elastizität der Qualität der elterlichen Fürsorge und der Fähigkeiten im frühen Lebenszyklus

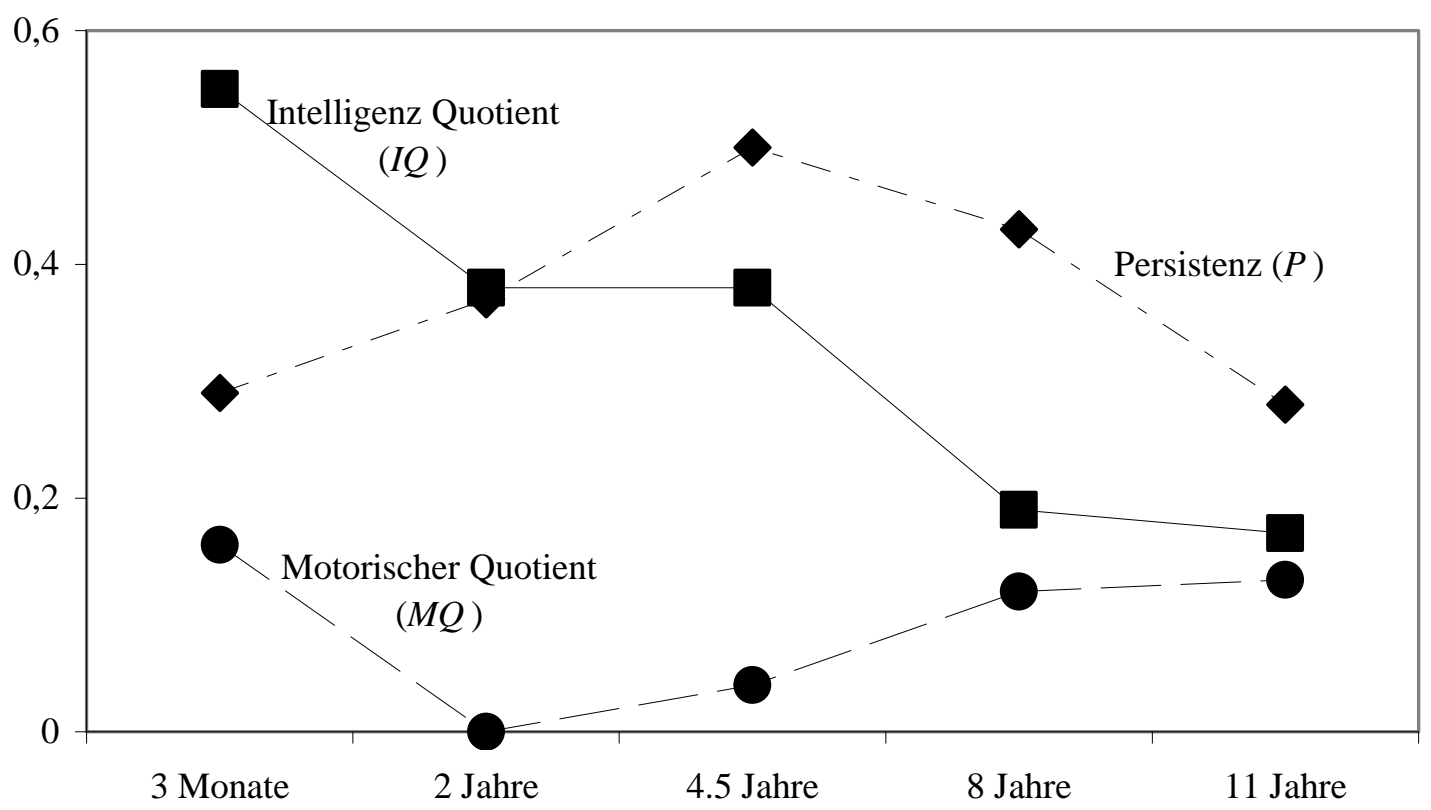

Werte entnommen aus Blomeyer et al. (2009, Table 2), eigene Darstellung. Für jede Fähigkeit und jede Periode wurde jeweils eine lineare Regressionsgleichung geschätzt, in der außer der Qualität der elterlichen Fürsorge die Fähigkeiten aus der Vorperiode enthalten sind. Da alle Größen in natürlichen Logarithmen eingehen, handelt es sich bei den Schätzwerten um Elastizitäten. Alle geschätzten Elastizitäten über 0,2 sind zum 5\% Niveau signifikant von null verschieden, alle Schätzwerte unter 0,2 sind statistisch nicht von null verschieden.

Die partielle Elastizität zwischen der Qualität der elterlichen Fürsorge und den motorischen Fähigkeiten fällt in allen Entwicklungsstufen positiv aus. Zum 5\% Niveau kann jedoch die Nullhypothese (der Wert ist nicht von null verschieden) nicht verworfen werden. Die partielle Elastizität zwischen der Qualität der elterlichen Fürsorge und den nichtkognitiven Fähigkeiten fällt in allen Entwicklungsstufen positiv aus 
und in allen Stufen kann die Nullhypothese zum 5\% Niveau verworfen werden. Demnach bleibt die elterliche Fürsorge ein wichtiger Input für die Fähigkeit, sich im Handlungsablauf nicht ablenken zu lassen, in allen Entwicklungsstufen. Die partielle Elastizität hat im Vorschulalter mit 0,5 den höchsten Wert, sinkt aber in keinem Alter unter den Wert von 0,3.

Gibt es Zusammenhänge zwischen den grundlegenden kognitiven, motorischen und nichtkognitiven Fähigkeiten und dem Schulerfolg? Blomeyer et al. (2008), können mit Hilfe des Längsschnittcharakters der Mannheimer Risikokinderstudie erstens zeigen, dass alle drei grundlegenden Fähigkeiten im Vorschulalter einen signifikanten Beitrag zur Erklärung der Schulnoten in Lesen, Schreiben und Rechnen im Alter von 8 Jahren haben. Den Schätzungen zu Folge führt ein um 1\% höherer $I Q(P)$ im Alter von 4,5 Jahren zu einer 0,84\% (0,32\%) höheren Note in Lesen (ähnlich bei den anderen Fächern). Zweitens haben Kinder mit höheren Werten in den drei grundlegenden Fähigkeiten im Schulalter, mit 8 Jahren, eine höhere Wahrscheinlichkeit ein Gymnasium aufzusuchen. Den Schätzungen zu Folge führt ein um 1\% höherer IQ $(M Q, P)$ mit 8 Jahren zu einer um 0,8\% (0,37\%, 0,49\%) höheren Wahrscheinlichkeit ein Gymnasium zu besuchen. Dies verdeutlicht das Ausmaß der Komplementaritäten in der Entwicklung zwischen Kindheit und Jugendalter. Mit höheren Fähigkeiten in der Kindheit sind deutlich bessere Schulleistungen verbunden.

\section{Bildungspolitische Alternativen}

Eine für die Bildungspolitik wichtige Frage betrifft den Zusammenhang zwischen der Höhe der sozio-emotionalen und den finanziellen Ressourcen, die den Kindern im Entwicklungsprozess zur Verfügung stehen, sowie ihrem Beitrag zur Entwicklung und zum Schulerfolg. Auf der Basis der Mannheimer Risikokinderstudie erhalten Blomeyer et al. (2009) drei Ergebnisse:

1. Die finanziellen Ressourcen haben für sich keinen weiteren Erklärungsbeitrag als Input in die Produktion von grundlegenden kognitiven und nichtkognitiven Fähigkeiten, wenn in der Analyse die sozio-emotionalen Ressourcen berücksichtigt werden. Im Kindesalter ist die Qualität der sozio-emotionalen Ressourcen, die elterliche Fürsorge, entscheidend, nicht die Höhe der finanziellen Ressourcen. Das (Klein-) Kind kann nicht unmittelbar von besseren materiellen Ressourcen profitieren, sondern nur von der Verfügbarkeit höherwertiger emotionaler Ressourcen.

2. Die Korrelation der beiden Arten von Ressourcen beträgt 0,1. Demnach ist in den Daten der Mannheimer Risikokinderstudie eine Verdopplung des Pro-KopfEinkommens der Familienmitglieder mit einer 10 prozentigen Zunahme der den Kindern zur Verfügung stehenden sozio-emotionalen Ressourcen verbunden.

3. 3. Die finanziellen Ressourcen sind beim Übergang auf eine Einrichtung mit höherwertiger sekundärer Bildung von Bedeutung. Eine Erhöhung des Pro-KopfFamilieneinkommens um 10\% erhöht bei ansonsten gleichen grundlegenden Fä- 
higkeiten der Kinder, die Wahrscheinlichkeit des Besuchs eines Gymnasiums um 1,8\%. Nach diesen Berechnungen spielt Geld für den Übergang ins Gymnasium demnach noch immer eine, wenngleich quantitativ eher bescheidene, Rolle.

Bei gleichen Fähigkeiten würden von 1.000 Kindern, die in um $10 \%$ ärmeren Haushalten leben, 18 weniger aufs Gymnasium gehen als in um 10\% reicheren Haushalten. Kinder, die das Potential haben, ein Gymnasium mit Erfolg abzuschließen, können dies aus Geldmangel nicht tun. Es gelingt Ihnen nicht, Kredite aufzunehmen, um sich weiter zu bilden. Kinder im Alter von 10 Jahren können sich noch nicht auf einem Kreditmarkt bewegen. Das Problem kann mit ausreichenden, einkommensabhängigen finanziellen Transfers gelöst werden, beispielsweise mit einem Gutschein im Falle des Besuchs eines Gymnasiums.

Ebenso wichtig für die Bildungspolitik sind die drei Worte „bei gleichen Fähigkeiten“. Die Berechnung trifft nur für den hypothetischen Fall der gleichen Fähigkeiten der Kinder in den relativ reicheren und den relativ ärmeren Haushalten zu. In der sozialen Realität weisen die Fähigkeiten der Kinder im Alter von 8 Jahren bereits erhebliche Unterschiede auf. Die Konsequenzen der Unterschiede in den Fähigkeiten können wiederum mit den auf Basis der Mannheimer Längsschnittdaten berechneten Werten verdeutlicht werden. Wenn der IQ bei einem Kind im Alter von 8 Jahren um $2 \%$ niedriger ist als bei einem anderen Kind, dass ansonsten in einer Familie mit gleichem Haushaltseinkommen lebt (und gleiche motorische und nichtkognitive Fähigkeiten hat), dann sinkt die Wahrscheinlichkeit ein Gymnasium zu besuchen, um 1,7\%. Bei gleichem Haushaltseinkommen und ansonsten gleichen Fähigkeiten werden von 1.000 Kindern, die im Alter von 8 Jahren einen um 2\% niedrigeren IQ haben, 17 weniger aufs Gymnasium gehen als von 1.000 Kindern, deren IQ um 2\% höher ist.

Da das Haushaltseinkommen für sich genommen keinen direkten Einfluss auf die Fähigkeitsentwicklung der Kinder hat, sondern nur indirekt, indem es mit einer höheren Qualität der elterlichen Fürsorge einhergeht, werden bildungspolitische Alternativen erkennbar. Falls es das Ziel der Bildungspolitik ist, den Anteil der Schüler, die ein Gymnasium besuchen, zu erhöhen, dann helfen Einkommenstransfers beim Übergang ins Gymnasium. Einkommenstransfers mildern die Konsequenzen von Unvollkommenheiten bei der Beleihung von Humankapital im Jugendalter. Falls es das Ziel der Bildungspolitik ist, die Fähigkeiten der Schüler im Alter von 8 Jahren zu verbessern, damit die Chancen steigen, durch verbesserte Fähigkeiten ein Gymnasium besuchen zu können, dann ist es notwendig, Kindern einen verbesserten $\mathrm{Zu}$ gang zu einer höherwertigen Qualität sozio-emotionaler Ressourcen in der Kindheit zu verschaffen. Wenn es beispielsweise gelingt, die Qualität sozio-emotionaler Ressourcen, die dem Kind zur Verfügung stehen, im Säuglingsalter um 1\% zu verbessern, steigt nach Blomeyer et al. (2009) der IQ (MQ, Persistenz) bis zum Alter von 8 Jahren um 0,96 (0,50, 0,55)\%. Damit erhöht sich die Wahrscheinlichkeit eines Gymnasiumsbesuchs um 1,2\% und zudem ist der IQ (MQ, Persistenz) im Alter von 
11 Jahren um 1,11 (0.56, 0,60)\% höher. Damit wird der Weg zum Gymnasium erleichtert. Angemessene Maßnahmen im vorschulischen Alter verbessern die Fähigkeiten. Damit wird der Weg zum Gymnasium erleichtert. Für Kinder aus ärmeren Haushalten sollten die frühkindliche sozio-emotionale und die materielle Förderung beim Übergang zum Gymnasium verbunden werden.

\section{Die Erträge von altersabhängigen Investitionen im Ar- beitsleben}

Die empirische Basis zur Bestimmung der lebenslangen Erträge zusätzlicher vorschulischer Bildungsinvestitionen ist aufgrund unvollständiger oder noch nicht vorhandener Längsschnittdaten, die auch das Arbeitsleben abdecken, noch eingeschränkt. Da über die langfristigen Erträge solcher Investitionen wenig quantitative Evidenz vorhanden ist, möchte ich in diesem Abschnitt ausgewählte Ergebnisse der Studie von Pfeiffer und Reuß (2008b) referieren. Der Humankapitalaufbau wird als Funktion von kognitiven und nichtkognitiven Fähigkeiten modelliert, wobei die frühe Ungleichheit in der Entwicklung von Fähigkeiten und die daraus resultierende Heterogenität der Erträge von zusätzlichen Bildungsinvestitionen im Vordergrund der Modellierung stehen.

Der Ertrag frühkindlicher Bildungsinvestitionen hängt von der Art, dem Umfang, dem Zeitpunkt und der Dauer dieser Anstrengungen ab. Zudem spielen die bereits vorhandenen Fähigkeiten der Kinder und die Qualität der familiären Fürsorge (siehe oben) für die Wirksamkeit weiterer Investitionen eine wichtige Rolle. Drittens können die Ziele von staatlichen (und privaten) Bildungsanstrengungen vielfältig sein, mit weiteren Konsequenzen auch für deren Erträge. So können staatliche Bildungsanstrengungen etwa dazu dienen, kompensierend benachteiligte Kinder zu fördern oder aber dazu, Kindern, die bereits von ihrem Familienhintergrund her eine gute Entwicklung aufweisen, weitere Impulse zu verschaffen, etwa zur Stärkung der sprachlichen oder sozialen Kompetenzen. Die optimale Förderung dieser beiden Zielgruppen wird sich im Hinblick auf den Mitteleinsatz unterscheiden. Die Festlegung von Zielen hängt von der relativen Gewichtung von Effizienz- und Gleichheitsüberlegungen ab (siehe dazu Pfeiffer und Reuß (2008a)). Wie Bildungsinvestitionen im Vorschulalter gestaltet sein müssen, damit sie den erwünschten lebenslangen Ertrag bringen, und wie viel dies kostet, kann durch Versuch und Irrtum bestimmt werden. Für die folgenden Ausführungen wird angenommen, dass es gelingt, effektive Maßnahmen einzusetzen.

Pfeiffer und Reuß (2008b) vergleichen die Erträge von zusätzlichen Investitionen in die Fähigkeiten von Kindern, die entweder im Vorschulalter (bis zum 6. Lebensjahr) oder im Alter zwischen 6 und 12 Jahren getätigt werden. Der Umfang beträgt jeweils 10\% der aktuellen jährlichen Pro-Kopf-Ausgaben für Kinder bis zum sechsten Lebensjahr (das sind 658,80 €), beziehungsweise 10\% der Ausgaben im Primärschul- 
bereich (das sind 428,50 €). Die Erträge werden in Form einer Erhöhung des Einkommens im Lebenszyklus modelliert, die an die empirische Verteilung der Arbeitsverdienste in Deutschland angepasst wird. Aufgrund des Fähigkeitsmultiplikators der frühen Kindheit übersteigen die zu erwartenden Erträge von Investitionen, die im richtigen Umfang und zur rechten Zeit getätigt werden, im Lebenszyklus die Kosten um ein Vielfaches. Nach Pfeiffer und Reuß (2008b) würde eine Erhöhung der Bildungsinvestitionen im Umfang von 658,80 € pro Kind pro Jahr bis zum 5. Lebensjahr das Lebenseinkommen (im Alter bis 65 Jahre) der geförderter Kinder um bis zu $55590 €$ erhöhen. Werden zusätzliche Mittel im Alter vom 6. bis zum 11. Lebensjahr investiert, wird ein Ertrag in Form eines höheren Lebenseinkommens von bis zu $37177 €$, oder 66\% des Ertrags der Investitionen im Vorschulalter, erzielt. Die Abschätzungen, die im Folgenden zusammengefasst werden, deuten darauf hin, dass die ertragreichere Politik zur Steigerung des Humankapitals und zur Reduktion von Ungleichheit im Vorschulalter ansetzen müsste. ${ }^{8}$ Dennoch kann die primäre Schulausbildung ebenfalls noch eine wichtige Rolle spielen.

Um Ungleichheit zu berücksichtigen und die Erträge zusätzlicher frühkindlicher Investitionen in Abhängigkeit von dieser Ungleichheit abzuschätzen, werden 4.432 Einzelbeobachtungen aus der Punkteverteilung des PISA-2000-Leseleistungstests deutscher Schüler ausgewertet. Aus diesen Beobachtungen werden sieben Perzentile für die Punktzahl des Leseleistungstests berechnet (Tabelle 3). Diese sieben Werte des Leseleistungstests werden im Modell durch Jahr für Jahr konstante, jedoch für jeden Wert charakteristische Bildungsinvestitionen in den Perioden 0 bis 18 erzeugt. Beispielsweise erhält der Schüler des 99ten Perzentil der PISA-Testverteilung ceteris paribus die 2,7684-fache Bildungsinvestition des Schülers des 50ten Perzentils (Tabelle 3, Spalte 3). Spalte 3 fasst das Ausmaß der Bildungsungleichheit in Deutschland zusammen.

Im Modell ist die Heterogenität des Arbeitsverdienstes ein Ergebnis der Ungleichheit in den Fähigkeiten und der Funktionsweise von Arbeitsmärkten. Der Ertrag zusätzlicher Bildungsinvestitionen wird durch die Veränderung des (abdiskontierten) Lebenseinkommens im Alter zwischen 18 und 65 Jahren geschätzt. Dieser wird den (aufdiskontierten) Kosten einer Bildungsinvestition gegenüber gestellt. Die Ausgaben pro Schüler in primären Bildungseinrichtungen beliefen sich im Jahre 2004 in Deutschland auf $4.285 €$, für Sekundarschulbildung auf $6.561 €$ und $10.613 €$ für tertiäre Bildung (OECD (2007)). Für die ersten sechs Jahre gibt es keine entsprechenden Zahlen. Stattdessen verwenden Pfeiffer und Reuß (2008b) für die Vorschulzeit die vom Statistischen Bundesamt geschätzten familiären Ausgaben für Kinder. Diese betrugen im Jahr 2003 im Durchschnitt $6.588 €$ jährlich (Statistisches Bundesamt 2006a). Das 10. Perzentil der Ausgabenverteilung betrug $3.900 €$ und das 90 . Perzentil $10.344 €$. Das entspricht einem Verhältnis von 2,65.

8 Die Folgen der altersabhängigen Humankapitalbildung für die lebenslange Ungleichheit und die Entwicklung von Arbeitsverdiensten, Bruttosozialprodukt und Renten werden in Pfeiffer und Reuß (2008c) analysiert. 
Tabelle 3: Perzentile der PISA 2000 Verteilung für Lesen, Investitionen

\begin{tabular}{ccc}
\hline Perzentil & PISA Lesepunktzahl & Investitionen (normiert) \\
\hline 1. & 236,57 & 0,01467 \\
10. & 362,70 & 0,2611 \\
25. & 438,95 & 0,5884 \\
50. & 507,77 & 1 \\
75. & 568,64 & 1,452 \\
90. & 619,80 & 1,8929 \\
99. & 707,23 & 2,7684 \\
\hline
\end{tabular}

Quelle: Pfeiffer und Reuß (2008b:36, Tabelle 1).

Nimmt man nun vereinfachend an, dass jährliche Ausgaben für ein Kind von $3.900 €$ dazu führen, dass das Kind das 10. Perzentil der PISA-Verteilung erreicht und beim Lebenseinkommen ebenso das 10. Perzentil erreicht usw., dann kann mit dem Simulationsmodell der Zusammenhang zwischen altersabhängigen Investitionen und dem Ertrag in Form eines höheren Lebenseinkommens abgeschätzt werden. Das Ergebnis wird in Tabelle 4 für das 10., 50., und das 90. Perzentil zusammengefasst.

Tabelle 4: Ausgaben für Kinder, PISA Leseleistung, und abdiskontiertes Lebenseinkommen

\begin{tabular}{cccc}
\hline \hline Perzentil & $\begin{array}{c}\text { Ausgaben für } \\
\text { Kinder / Jahr }\end{array}$ & $\begin{array}{c}\text { PISA Punktzahl } \\
\text { Lesen }\end{array}$ & $\begin{array}{c}\text { Abdiskontiertes } \\
\text { Lebenseinkommen } \\
\text { (Alter: 18 Jahre) }\end{array}$ \\
\hline 10. & $3900 €$ & 362.7 & $411956 €$ \\
50. & $6588 €$ & 507.77 & $821274 €$ \\
90. & $10344 €$ & 619.8 & $1239000 €$ \\
\hline \hline
\end{tabular}

Werte entnommen aus Pfeiffer und Reuß (2008b:37, Tabelle 2).

Welche lebenslangen (erwarteten) Erträge haben zusätzliche frühkindliche Bildungsinvestitionen in Abhängigkeit von der Ungleichheit der vorhandenen Investitionen? Im Sinne eines Gedankenexperiments (siehe Tabelle 5) werden die Bildungsinvestitionen für die drei Kinder, die von ihrem sozioökonomischen Hintergrund her so viele Investitionen in ihre Fähigkeiten erhalten, dass ihre Leseleistung im Alter von 16 Jahren dem 10., 50. und 90. Perzentil der PISA-Verteilung entspricht, stellvertretend um 658,80 € pro Jahr für die ersten sechs Lebensjahre erhöht. 658,80 € entsprechen 
dem Wert einer zehnprozentigen Erhöhung der durchschnittlichen Ausgaben für Kinder. Pro Jahr würden demnach für jedes Kind weitere 658,8 $€$ in seine Fähigkeiten investiert. Dies entspricht bei etwa 650.000 Geburten im Jahre 2008 einer Summe von $428220000 €$ für eine Geburtskohorte. Es wird angenommen, dass diese zusätzlichen Mittel vollständig in zusätzliche Bildungsinvestitionen transformiert werden. Dabei kann es sich um einen direkten Transfer zu dem Kind in der Familie oder um eine Förderung in einer Bildungseinrichtung handeln.

Tabelle 5 fasst die Ergebnisse der Erträge zusätzlicher Bildungsinvestitionen sowie die zugehörigen Kosten zusammen. Der zusätzliche Nutzen einer Erhöhung der Investitionen nimmt mit den bereits vorhandenen Investitionen ab. So führt eine Erhöhung um 658,80 € zu einem Anstieg der Bildungsinvestition um 0.181 Punkte für das 10. Perzentil, 0.157 Punkte für das 50. Perzentil und 0.132 Punkte für das 90. Perzentil. Für benachteiligte Kinder nehmen die Bildungsinvestitionen daher relativ stärker zu. Während die Kosten für alle Individuen aus den sieben Perzentilen gleich sind, sind die Erträge unterschiedlich. Die in absoluten Geldgrößen gemessenen Erträge bewegen sich zwischen $32.000 €$ (90. Perzentil) und 55.590 € (10. Perzentil). Die Ergebnisse verdeutlichen, dass der Ertrag den Aufwand für alle Perzentile um ein vielfaches übersteigt. Der interne Zinsfuß liegt zwischen 595 und 1.033\%. Bei einem Zinsfuß von 1.033\% (10. Perzentil) verzehnfacht sich jeder investierte EURO über den gesamten Lebenszyklus. Das Lebenseinkommen nimmt zwischen 2,58 und $13,49 \% \mathrm{zu}$.

Tabelle 5: Altersabhängige Erhöhung von Bildungsinvestitionen und die Zunahme des Humankapitals im Lebenszyklus

\begin{tabular}{ccccc}
\hline \hline $\begin{array}{c}\text { Perzentil } \\
\text { der PISA } \\
\text { Verteilung }\end{array}$ & $\begin{array}{c}\text { Bildungskosten } \\
\text { aufdiskontiert } \\
\text { zum Alter von 18 } \\
\text { Jahren }\end{array}$ & $\begin{array}{c}\text { Veränderung des } \\
\text { abdiskontierten } \\
\text { Lebenseinkom- } \\
\text { men zum Alter } \\
\text { von 18 Jahren }\end{array}$ & $\begin{array}{c}\text { Prozentualer An- } \\
\text { stieg des Lebens- } \\
\text { einkommens }\end{array}$ & $\begin{array}{c}\text { Interner } \\
\text { Zinsfuß der zu- } \\
\text { sätzlichen Bil- } \\
\text { dungsinvestition }\end{array}$ \\
\hline \multicolumn{7}{c}{$10 \%$ mehr Bildungsinvestitionen im (Vorschul-)Alter zwischen 0 und 5 Jahren } \\
\hline 10. & $5378 €$ & $55590 €$ & $+13,49 \%$ & $1033,73 \%$ \\
50. & $5378 €$ & $38177 €$ & $+4,65 \%$ & $709,93 \%$ \\
90. & $5378 €$ & $32000 €$ & $+2,58 \%$ & $595,06 \%$ \\
\hline $10 \%$ mehr Bildungsinvestitionen im (Schul-)Alter zwischen 6 und 11 Jahren \\
\hline 50. & $4775 €$ & $37177 €$ & $+9,02 \%$ & $778,55 \%$ \\
90. & $4775 €$ & $26221 €$ & $+3,19 \%$ & $549,11 \%$ \\
\hline \hline
\end{tabular}

Werte entnommen aus: Pfeiffer und Reuß (2008b:38, 39, Tabelle 3, Tabelle 4). 
Führt man die zusätzlichen Investitionen nicht in den ersten 6 Jahren, sondern im Alter von 6 bis 12 durch, so ist die Steigerung des Lebenseinkommens geringer, jedoch immer noch höher als die Kosten (Tabelle 5). Der Umfang der Investitionen beträgt in diesem Gedankenexperiment 10\% der Ausgaben im Primärschulbereich (das sind 428,50 €). Daraus wird ein höheres Lebenseinkommens von bis zu 37177 $€$, oder 66\% des Ertrags der Investitionen im Vorschulalter, erzielt. Der interne Zinsfuß variiert zwischen 439 und $778 \%$.

\section{Abschließende Bemerkungen}

In dem vorliegenden Beitrag wird argumentiert, dass in der sozialen Realität die Bildungsungleichheit im Vorschulalter eine der wichtigsten Ursachen für die Ungleichheit von Fähigkeiten und Kompetenzen auch im Schulalter und darüber hinaus ist. Bereits in der frühen Kindheit werden, überwiegend in der Familie, die Kapazitäten aufgebaut (oder nicht aufgebaut), welche eine nachhaltige Voraussetzung für den Schul- und Arbeitsmarkterfolg schaffen. Die Unterschiede in der Verfügbarkeit insbesondere von sozio-emotionalen Ressourcen in der (frühen) Kindheit und davor entfalten eine prägende Kraft, die später, im Schulalter, in der sozialen Wirklichkeit kaum mehr aufgeholt werden kann. Im Schulalter kommen finanzielle Restriktionen durch Armut in der Familie noch hinzu.

Schulversagen, Jugendarbeitslosigkeit, Desintegrationstendenzen bereits unter Jugendlichen sowie eine Zunahme der Ungleichheit der Arbeitsverdienste (unter anderem Gernandt und Pfeiffer (2007)) lassen vermuten, dass in Deutschland zu wenig in die Entwicklung von Fähigkeiten im frühen Kindesalter investiert wird. Für benachteiligte Kinder hat die Bildungsungleichheit vor dem Schulalter auch in den industrialisierten Ländern trotz Schulpflicht, hohen Bildungsausgaben und wirtschaftlichem Wohlstand ihren vielfach schicksalhaften Charakter noch nicht verloren. Neben dem erheblichen individuellen Leid, das mit diesem Schicksal verbunden ist, entstehen der Gesellschaft dadurch signifikante Kosten, unter anderem im Gesundheits- und Sozialsystem. Medizinische Forschungen deuten beispielsweise darauf hin, dass Vernachlässigung und Gewalt in der Kindheit die Anfälligkeit für Depressionen, aber auch für Infektionskrankheiten im Erwachsenenalter erhöhen.

Um dies zu ändern, bleibt es eine vordringliche Aufgabe auch der Bildungspolitik, den Zugang zu einer angemessenen emotionalen Fürsorge von Anfang an weiter zu verbessern. Darüber hinaus ist es notwendig, den betroffenen Kindern bis ins Jugendalter altersgemäß und individuell zur Seite zu stehen. Bei den vielfältigen Bemühungen und Programmen zur Förderung im Vorschulalter wird die Notwendigkeit einer Förderung darüber hinaus oftmals häufig übersehen. Für Jugendliche und Erwachsene, die während der Kindheit nur unzureichend gefördert wurden, sollten Maßnahmen erforscht werden, die helfen können, vielfältige Benachteiligungen aus der Kindheit im späteren Lebenszyklus zu bewältigen. 


\section{Literatur}

Ainslie, George (2001): Breakdown of Will. New York: Cambridge University Press.

Amor, David J. (2003): Maximizing Intelligence. New Brunswick. Transaction Publishers.

Apolte, Thomas/Funcke, Antje (Hrsg.) (2008): Frühkindliche Bildung und Betreuung. Reformen aus ökonomischer, pädagogischer und psychologischer Perspektive. Baden-Baden: Nomos.

Beck, Bärbel/Klieme, Eckhart (Hrsg.) (2006): Sprachliche Kompetenzen. Konzepte und Messung. Weinheim: Beltz Pädagogik.

Beckett, Celia/Maughan, Barbara / Rutter, Michael/Castle, Jenny/Colvert, Emma/Groothues, Christine/Kreppner, Jana/Stevens, Suzanne/O'Connor, Thomas/Sonuga-Barke, Edmund (2006): Do the Effects of Early Severe Deprivation on Cognition Persist Into Early Adolescence? Findings from the English and Romanian Adoptees Study. In: Child Development, Jg. 77, H. 3, 696-711.

Benabou, Roland/Tirole, Jean (2004): Willpower and Personal Rules. In: Journal of Political Economy, Jg. 112, H. 4, 848-887.

Black, Sandra E./Devereux, Paul J./Salvanes, Kjell (2007): From the Cradle to the Labor Market? The Effect of Birth Weight on Adult Outcomes. In: The Quarterly Journal of Economics, Jg. 122, H. 1, 409-39.

Blomeyer, Dorothea/Coneus, Katja/Laucht, Manfred/Pfeiffer, Friedhelm (2009): Initial Risk Matrix, Home Resources, Ability Development and Children's Achievement. In: Journal of the European Economic Association, P\&P Jg. 7, H. 2-3, 1-11.

Blomeyer, Dorothea/Coneus, Katja/Laucht, Manfred/Pfeiffer, Friedhelm (2008): Self-productivity and Complementarities in Human Development: Evidence from the Mannheim Study of Children at Risk. Mannheim: ZEW Discussion Paper No. 08-067.

Braun, Katharina/Stern, Elsbeth (2007): Neurowissenschaftliche Aspekte der Erziehung, Bildung und Betreuung von Kleinkindern. Expertise für die Enquetekommission Chance für Kinder des Nordrheinwestfälischen Parlaments.

Coneus, Katja/ Spiess, Katharina (2008): The Intergenerational Transmission of Health in Early Childhood, Mannheim: ZEW Discussion Paper No. 08-073.

Ciccone, Antonio/Peri, Giovanni (2006): Identifying Human-Capital Externalities: Theory with Applications. In: Review of Economic Studies, Jg. 73, H. 2, 381412. 
Cunha, Flavio/Heckman, James J. (2007): The Technology of Skill Formation. In: The American Economic Review, Jg. 97, H. 2, 31-47.

Cunha, Flavio/Heckman, James J. (2009): The Economics and Psychology of Inequality and Human Development. Cambridge, MA: NBER Working Paper 14695.

Danese, Andrea/Pariante, Carmine M./Caspi, Avshalom/Taylor, Alan/Poulton, Richie (2007): Childhood Maltreatment Predicts Adult Inflammation in a Lifecourse Study. In: Proceedings of the National Academy of Sciences, Jg. 104, H. 4, 1319-1324.

Erikson, Erik (1959): Identity and the Life Cycle. In: Psychological Issues, Jg. 1, H. 1.

Gebel, Michael/Pfeiffer, Friedhelm (2009): Educational Expansion and its Heterogeneous Returns for Wage Workers. In: Schmollers Jahrbuch - Journal of the Social Sciences (erscheint demnächst).

Gernandt, Johannes/Pfeiffer, Friedhelm (2007): Rising Wage Inequality in Germany. In: Jahrbücher für Nationalökonomie und Statistik, Jg. 227, H. 4, 358-380.

Gollwitzer, Peter M. (1999): Implementation Intentions: Strong Effects of Simple Plans. In: American Psychologist, Jg. 54 H. 7, 493-503.

Gollwitzer, Peter M./Gawrilow, Caterina/Oettingen, Gabriele (2008): The Power of Planning: Effective Self-Regulation of Goal Striving. New York University: Manuscript.

Hanushek, Eric A./Wößmann, Ludger (2008), The Role of Cognitive Skills in Economic Development. In: Journal of Economic Literature, Jg. 46, H. 3, 607668.

Heckhausen, Jutta/Heckhausen, Heinz (2006): Motivation und Entwicklung. In: Heckhausen, Jutta/Heckhausen, Heinz (Hrsg.): Motivation und Handeln. Berlin: Springer, 393-454.

Heckman, James J. (2000): Policies to Foster Human Capital. In: Research in Economics, Jg. 54, H. 1, 3-56.

Heckman, James J. (2007): The Economics, Technology and Neuroscience of Human Capability Formation. In: Proceedings of the National Academy of Sciences, Jg. 104, H. 3, 132250-5.

Heckman, James J. (2008): Skills, Schools and Synapses. In: Economic Inquiry, Jg. 46, H. 4, 289-324.

Holodynski, Manfred/Stallmann, Freia/Seeger, Dorothee (2008): Entwicklung als soziokultureller Lernprozess: Die Bildungsbedeutung von Bezugspersonen für Kinder. In: Apolte, Thomas/Funcke, Antje (Hrsg.), 91-129. 
Kramarz, Francis/Machin, Stephen/Ouazad, Amine (2008): What Makes a Test Score? The Respective Contributions of Pupils, Schools, and Peers in Achievement in English Primary Education. Bonn: IZA Discussion Paper No. 3866.

Laucht, Manfred (2005): Die langfristigen Folgen früher Entwicklungsrisiken: Ergebnisse der Mannheimer Längsschnittstudie zu Risiko- und Schutzfaktoren. In: Arnoldy, Peter/Traub, Birgit (Hrsg.): Sprachentwicklungsstörungen früh erkennen und behandeln. Karlsruhe: Loeper, 169-183.

Laucht, Manfred/Esser, Günter/Schmidt, Martin H. (1997): Developmental Outcome of Infants Born with Biological and Psychosocial Risks. In: Journal of Child Psychology and Psychiatry, Jg. 38, H. 7, 843-54.

Laucht, Manfred/Schmidt, Martin/Esser, Günter (2004): The development of at-risk children in early life. In: Educational and Child Psychology, Jg. 21, H. 1, 2031.

Ludwig, Volker/Pfeiffer, Friedhelm (2006): Abschreibungsraten allgemeiner und beruflicher Ausbildungsinhalte - empirische Evidenz auf Basis subjektiver Einschätzungen. In: Jahrbücher für Nationalökonomie und Statistik, Jg. 226, H. 3, 260-284.

Maier, Michael/Pfeiffer, Friedhelm/Pohlmeier, Winfried (2004): Overeducation and Individual Heterogeneity. In: Büchel, Felix/de Grip, Andries/ Mertens, Antje (Hrsg.): Overeducation in Europe: Current Issues in Theory and Policy. Cheltenham: Edward Elgar, 133-152.

Moretti, Enrico (2004): Workers' Education, Spillovers, and Productivity: Evidence from Plant-Level Production Functions. In: American Economic Review, Jg. 94, H. 3, 656-690.

Mühlenweg, Andrea (2008): Educational Effects of Alternative Secondary School Tracking Regimes in Germany. In: Schmollers Jahrbuch - Journal of Applied Social Science Studies, Jg. 128, H. 3, 351-379.

Mühlenweg, Andrea/Puhani, Patrick (2009): The Evolution of the School Entry Age Effect in a School Tracking System. In: Journal of Human Resources, forthcoming.

O’Connell, Michael/Sheikh, Hammad (2008): Achievement-related Attitudes and the Fate of "At-risk" Groups in Society. In: Journal of Economic Psychology, Jg. 29, H. 4, 508-21.

Pfeiffer, Friedhelm/Reuß, Karsten (2008a): Age-Dependent Skill Formation and Returns to Education. In: Labour Economics, Jg. 15, H. 4, 631-646.

Pfeiffer, Friedhelm/Reuß, Karsten (2008b): Ungleichheit und die differentiellen Erträge frühkindlicher Bildungsinvestitionen im Lebenszyklus. In: Apolte, Thomas/Funcke, Antje (Hrsg.), 25-43. 
Pfeiffer, Friedhelm/Reuß, Karsten (2008c): Intra- und intergenerationale Umverteilungseffekte in der bundesdeutschen Alterssicherung auf Basis humankapitaltheoretischer Überlegungen, In: Deutsche Rentenversicherung, Jg. 63, H. 1, 60 -84 .

Sodian, Beate/Koerber, Susanne (2008): Grundlagen für lebenslanges Lernen in den ersten Lebensjahren. In: Apolte, Thomas/Funcke, Antje (Hrsg.), 83-89.

Van den Berg, Gerard/Lindeboom, Maarten/Portrait, France (2006): Economic Conditions Early in Life and Individual Mortality. In: The American Economic Review Jg. 96, H. 1, 290-302.

Wells, Jonathan (2003): Parent-Offspring Conflict Theory, Signaling of Need, and Weight Gain in Early Life. In: The Quarterly Review of Biology, Jg. 78, H. 2, 169-202. 